Hautarzt 2020 $71: 737-738$

https://doi.org/10.1007/s00105-020-04690-4 Online publiziert: 17 . September 2020 (c) Springer Medizin Verlag GmbH, ein Teil von Springer Nature 2020

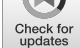

Julie Kind' $\cdot$ Michael Jünger ${ }^{2}$ Alexander Kapp ${ }^{3}$. Roland Kaufmann ${ }^{4}$. Jean Krutmann ${ }^{5} \cdot$ Hans F. Merk ${ }^{6}$. Thomas Ruzicka ${ }^{7}$ für die Schriftleitung Der Hautarzt

' Redaktion Fachzeitschriften Medizin, Springer Medizin Verlag GmbH, Heidelberg, Deutschland ${ }^{2}$ Klinik und Poliklinik für Hautkrankheiten, Universitätsmedizin Greifswald, Greifswald, Deutschland

${ }^{3}$ Klinik für Dermatologie, Allergologie und Venerologie, Medizinische Hochschule Hannover, Hannover, Deutschland

${ }^{4}$ Klinik für Dermatologie, Venerologie und Allergologie, Universitätsklinik Frankfurt, Frankfurt am Main, Deutschland

${ }^{5}$ IUF - Leibniz-Institut für Umweltmedizinische Forschung, Düsseldorf, Deutschland

${ }^{6}$ Klinik für Dermatologie und Allergologie, RWTH Aachen University, Aachen, Deutschland

${ }^{7}$ Klinik für Dermatologie und Allergologie, Ludwig-Maximilians-Universität München, München, Deutschland

\title{
Dank an Herrn Professor Michael Meurer
}

\section{Schriftleitung und Verlag danken dem langjährigen Schriftleiter der Zeitschrift Der Hautarzt}

Nach 18 Jahren legt Herr Prof. Michael Meurer (• Abb. 1) zum 01.10.2020 sein Amt als Schriftleiter und CME-Rubrikherausgeber der Zeitschrift Der Hautarzt nieder.

Mit der Umstrukturierung der Zeitschrift 2002 hat sich das Herausgebergremium neu aufgestellt, und das Team der sieben Schriftleiter hat in den Folgejahren das Gesicht der Zeitschrift und ihre Ausrichtung geprägt. Das Schriftleitergremium aus Herrn Prof. Michael Jünger, Herrn Prof. Alexander Kapp, Herrn Prof. Roland Kaufmann (seit 2010), Herrn Prof. Jean Krutmann, Herrn Prof. Hans F. Merk, Herrn Prof. Michael Meurer, Herrn Prof. Thomas Ruzicka und Herrn Prof. Wolfgang Tilgen (bis 2009) hat dafür Sorge getragen, dass Der Hautarzt seine Position als ein bedeutendes Kommunikationsforum für die deutschsprachige Dermatologie ausbauen konnte und das Profil als wichtige, gern gelesene Fort- und Weiterbildungszeitschrift u. a. mit den Leitthemenheften weiter geschärft wurde.

Im Jubiläums-Editorial 2009 schrieb Michael Meurer: „Es erschien uns allen von Anfang an wichtig, die von Alfred Marchionini begründete und von großen dermatologischen Lehrern, wie Otto Braun-Falco, Gerd Plewig, Günther Burg, Detlef Petzoldt, Urs Schnyder, Theo Nasemann, Klaus Wolff, Eva-Bettina Bröcker und Peter Fritsch, souverän weitergeführte Tradition von Der Hautarzt in erneuerter, den aktuellen Bedürfnissen der Fort- und Weiterbildung unseres Fachbereiches angepassten Form fortzuführen. “ [1] Dies ist dem siebenköpfigen Schriftleiterteam zweifellos gelungen.

Michael Meurer, geborener Dresdener, studierte in Erlangen-Nürnberg, Innsbruck, Heidelberg und München Humanmedizin. Nach Stationen in der Inneren Medizin und der Chirurgie begann er seine dermatologische Laufbahn als Stipendiat der DFG (Deutsche Forschungsgemeinschaft) an der Mayo Clinic in Rochester und im Department of Dermatology, New York University Medical Center. Nach langjähriger Tätigkeit als Hautarzt und Hochschullehrer an der Dermatologischen Klinik und Poliklinik der LMU München unter Otto Braun-Falco erhielt er 1997 den
Ruf auf den Lehrstuhl für Dermatologie an der Medizinischen Fakultät Carl Gustav Carus der TU Dresden und leitete die Klinik bis 2009, das von ihm gegründete interdisziplinäre UniversitätsAllergiezentrum bis 2012. Er ist weiterhin als vorsitzender Stiftungsvorstand der Stiftung zur Förderung der Hochschulmedizin Dresden aktiv und arbeitet regelmäßig als Dermatologe in Dubai.

Der Springer-Verlag und das Redaktionsteam der Zeitschrift danken Michael

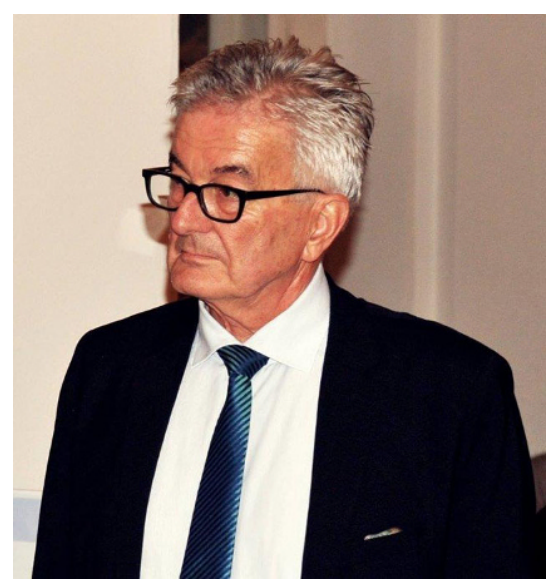

Abb. 1 ^ Prof. Dr. Michael Meurer 
Meurer sehr herzlich für die vertrauensvolle Zusammenarbeit, die immer von Wertschätzung geprägt war. In den 18 Jahren seiner Tätigkeit hat er nicht nur zahlreiche Artikel verfasst und begutachtet, sondern als Heftherausgeber auch eine Vielzahl von Leitthemenheften koordiniert und in der Amtszeit als CME-Rubrikherausgeber sage und schreibe 212 CME-Beiträge betreut. Er hat seine Funktion als Schriftleiter stets mit Freude, Stil, Objektivität und großer Zuverlässigkeit ausgeführt. Bat man ihn kurzfristig um Rat, konnten seine Schriftleiterkollegen und die Redaktion immer auf seine kritische, wohlüberlegte Einschätzung setzen. Er rettete manchen CME-Beitrag und auch manches Leitthemenheft, wenn ein Autor kurzfristig absprang.

Der dermatologische Nachwuchs lag Michael Meurer sehr am Herzen, sodass er die Rubrik „CME. Zertifizierte Fortbildung" von Beginn an mit viel Engagement und Weitblick betreute und die Zielsetzung der Rubrik nie aus den Augen verlor. Sein großartiges Netzwerk und sein breit aufgestelltes Wissen waren hier von unschätzbarem Wert. Auch bei den Sonderheften „FacharztTraining Dermatologie“ musste man Michael Meurer nicht lange bitten: Er war sofort von dem Konzept begeistert und als Autor im Boot. Dabei konnte man sicher sein, dass seine Beiträge stets hervorragend geschrieben und vorbereitet waren.

Die Schriftleitung, das Herausgeberboard und der Verlag danken Herrn Prof. Meurer für die jahrelange erfolgreiche, bereichernde Zusammenarbeit und für seine Verdienste um die Zeitschrift.

Mit den besten Wünschen für die kommenden Jahre

Schriftleitung und CME-Rubrikherausgeber Der Hautarzt

Redaktion Der Hautarzt

\section{Korrespondenzadresse}

\section{Dr. med. Julie Kind}

Redaktion Fachzeitschriften Medizin, Springer

Medizin Verlag GmbH

Tiergartenstr. 17, 69121 Heidelberg,

Deutschland

julie.kind@springer.com

Interessenkonflikt. J. Kind, M. Jünger, A. Kapp, R. Kaufmann, J. Krutmann, H.F. Merk und T. Ruzicka geben an, dass kein Interessenkonflikt besteht.

\section{Literatur}

1. Der Hautarzt im 60. Jahrgang. Die Schriftleitung gratuliert zum Jubiläum (2009). Hautarzt 60: 264-266; DOI https://doi.org/10.1007/s00105009-1763-8, Springer Medizin Verlag

\section{Derma Update Livestream: Innovativ und flexibel}

13. und 14. November aus Berlin

27. und 28. November aus Mainz

Umfangreiches Update-Wissen in schwierigen Zeiten: Erstmals können sich interessierte Ärzte für das komplette Dermatologie-Update-Seminar zum innovativen Livestream anmelden. Seien Sie LIVE dabei, bequem von zu Hause oder aus der Klinik! Die Interaktivität wird durch einen Livechat ermöglicht und die Zertifizierung ist beantragt, sodass Sie auch bei virtueller Teilnahme Ihre wichtigen CME-Punkte erhalten.

Das Update-Konzept: Die wichtigsten

Neuerungen des vergangenen Jahres aus der Dermatologie werden unter der wissenschaftlichen Leitung von Prof. Dr. Thomas Schwarz (Kiel) und Prof. Dr. Thomas Werfel (Hannover) kritisch selektiert, analysiert und zusammengefasst. Die Relevanz für den Klinik- und Praxisalltag der Ärzte steht dabei im Vordergrund und der ausführlichen Diskussion über den Livechat mit den Referenten wird viel Raum gegeben.

In diesem Jahr werden neben den Kerngebieten der Dermatologie die Hot Topics »Bakteriologie«, "Lichenoide und granulomatöse Dermatosen« und »Pruritus und Prurigoerkrankungen« vorgestellt.

Zum Gesamtpaket der Teilnahme gehören neben den umfangreichen Seminarunterlagen als eBook der Download aller Vortragspräsentationen und die Vorträge im Nachgang als Video-on-Demand auf der Videoplattform »streamed-up.com«.

Weitere Informationen zu Programm, Referenten und Anmeldung: derma-update.com

Veranstalter: med update $\mathrm{GmbH}$ Hagenauer Straße 53 65203 Wiesbaden 